\title{
Alternativas de cierre de fasciotomías en extremidades
}

\author{
Juan Pablo Henríquez R. ${ }^{1}$, Gonzalo Yáñez C. ${ }^{1}$ y Susana Searle F. ${ }^{2}$
}

\begin{abstract}
Alternatives for the closure of extremity fasciotomy
Fasciotomy is the mainstay of treatment and prevention of acute compartment syndrome. Given the important deep tissue edema, closure of the resulting wound generates a significant reconstructive challenge for the surgeon. The aim of this article is to provide an update concerning alternatives for closure of fasciotomy of limbs, for which a search of articles indexed in PubMed, Scielo and Epistemonikos databases was performed. At least 6 techniques were found, each of them with specific advantages and disadvantages. We recommend that the choice should be according to the surgeons experience, resources and context of each patient.
\end{abstract}

Key words: fasciotomy; compartment syndromes; wound closure techniques; extremities.

\section{Resumen}

La fasciotomía es el pilar del tratamiento y prevención del síndrome compartimental agudo. Una vez resuelto el cuadro agudo que derivó en la necesidad de ésta, el cierre de la herida resultante genera un importante desafío reconstructivo para el cirujano dado el importante edema residual de los tejidos. El objetivo de este artículo es entregar una actualización respecto a las alternativas de cierre de una fasciotomía de extremidades, para lo cual se realizó una búsqueda de artículos indexados en PubMed, Epistemonikos y Scielo. Se encontraron al menos 6 técnicas disponibles, cada una de ellas con determinadas ventajas y desventajas. Recomendamos que la elección sea de acuerdo a la experiencia del cirujano, los recursos disponibles y el contexto de cada paciente.

Palabras clave: fasciotomía; cierre de heridas; síndrome compartimental; extremidades.

\section{Introducción}

La fasciotomía es el pilar del tratamiento y prevención del síndrome compartimental agudo (SCA). Sin embargo, una vez resuelta la causa que produjo o condujo a la necesidad de la liberación del compartimiento, la herida resultante prolonga de forma significativa la estadía hospitalaria y su cierre genera un gran desafío reconstructivo ${ }^{1}$. En la literatura internacional se estima una incidencia anual de SCA en una extremidad traumatizada de 7,3 por 100.000 hombres y 7,0 por 100.000 mujeres $^{2}$. El SCA consiste en el aumento progresivo de la presión dentro de un compartimento osteofascial cerrado, que sobrepasa en primer lugar la presión venosa, causando gran edema, lo que contribuye finalmente a sobrepasar la presión arterial de perfusión de la extremidad, generando isquemia de los tejidos que de no ser tratada oportunamente evoluciona hacia la necrosis, pérdida de la extremidad y en raros casos, la muer- te ${ }^{3}$. Schwartz et al. reportan una mortalidad de un $47 \%$ posterior a un SCA de muslo ${ }^{4}$. En una revisión de casos médico-legales en Canadá, se reportaron 91 juicios específicos por SCA entre 1998 y $2008 . .^{5}$

El desafío del cierre de la herida radica en que los tejidos presentan gran edema, tanto superficial como profundo y la piel está sometida a gran tensión. El objetivo de este trabajo es realizar una revisión bibliográfica de las diferentes alternativas de cierre de las fasciotomías en extremidades y entregar una actualización que permita al cirujano optar por la mejor técnica de acuerdo al paciente.

\section{Materiales y Métodos}

Se realizó una búsqueda bibliográfica independiente por dos de los autores (JPH/GY) en los buscadores Pubmed, Epistemonikos y Scielo, incluyendo trabajos publicados hasta enero de 2017. Los criterios de selección fueron en primer lugar
Residente Cirugía General. Pontificia Universidad Católica de Chile.

${ }^{2}$ Profesor Asistente Adjunto. Sección de Cirugía Plástica y Reconstructiva, División de Cirugía.

Pontificia Universidad Católica de Chile. Santiago, Chile.

Recibido el 2 de agosto de 2017 y aceptado para publicación el 24 de octubre de 2017.

\section{Correspondencia a:} Dr. Gonzalo Yáñez Caro División de Cirugía. Hospital Clínico UC.

Diagonal Paraguay 362 $3^{\text {er }}$ piso. Santiago, Chile. gtyanez@gmail.com 
Tabla 1. Criterios de inclusión y exclusión

\begin{tabular}{|lll|}
\hline Criterio Evaluado & Inclusión & Exclusión \\
\hline Indicación de fasciotomía & $\begin{array}{l}\text { Síndrome compartimental agudo, síndrome compartimental crónico, } \\
\text { fasciotomía profiláctica por isquemia }\end{array}$ & Enfermedad de Dupuytren, úlceras \\
\hline Sitio de fasciotomía & Extremidades superior e inferior & Cuello, abdomen \\
Participante & Humanos & Animales, cadáveres \\
\hline
\end{tabular}

estudios realizados en humanos y que describieran alguna técnica de cierre de fasciotomías. Respecto a la indicación de la fasciotomía, sólo se incluyeron trabajos sobre SCA, síndrome compartimental crónico e isquemia con indicación profiláctica previo a reperfusión, excluyendo aquellos artículos relacionados a enfermedad de Dupuytren y úlceras (Tabla 1). En relación al sitio anatómico, se analizaron estudios sobre extremidades superiores e inferiores, eliminando del análisis fasciotomías en cuello y abdomen. Se excluyeron, trabajos en modelos animales y cadáveres. No se encontraron discordancias en cuanto a los artículos seleccionados entre los revisores.

La búsqueda en Pubmed se realizó mediante el glosario de términos $\mathrm{MeSH}$ indexados como "Fasciotomy", "Wound Closure Techniques", "Negative-Pressure Wound Therapy" y "extremities". Se agregaron, además, los sinónimos no indexados

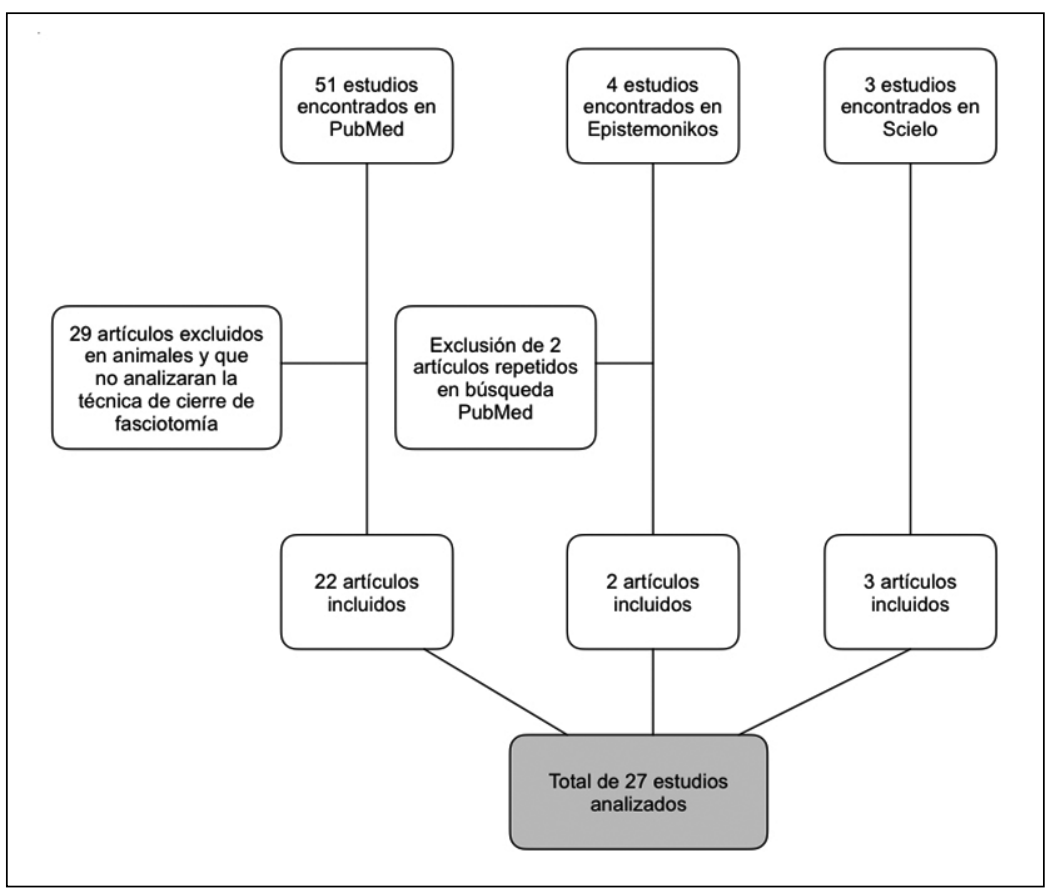

Figura 1. Diagrama de flujo. atingentes, quedando la búsqueda de la siguiente forma: "Fasciotomy" [Mesh] AND ("Wound Closure Techniques" [Mesh] OR "Negative-Pressure Wound Therapy" [Mesh] OR "Vacuum-Assisted Closure"[All Fields]) AND ("extremities" [MeSH Terms] OR "extremities" [All Fields] OR "limb" [All Fields] OR "limbs" [All Fields] OR "leg" [All Fields] OR "legs" [All Fields]). Se encontró un total de 51 artículos. Al aplicar los criterios de inclusión y exclusión anteriormente mencionados la búsqueda se redujo a 22 artículos (Figura 1). Entre estos, se encontró un review de Kakagia et al. de $2014^{6}$ sobre alternativas de cierre de fasciotomía, cuya subdivisión fue utilizada como base para el presente artículo, agregando de forma complementaria otras técnicas no mencionadas. El resto de los artículos $^{1,5,7,8-25}$ fueron revisados exhaustivamente.

A continuación, se realizó una búsqueda avanzada en Epistemonikos para abstracts que incluyeran "Fasciotomy Wound Closure". Se encontraron 4 artículos, dos de estos se repetían en la búsqueda de PubMed ${ }^{6,14}$. Los dos artículos restantes, de tipo revisión sistemática, fueron agregados al análisis ${ }^{26,27}$. Por último, en la base de datos Scielo se realizó la búsqueda en español con los términos "Cierre Fasciotomía” encontrando 3 artículos adicionales ${ }^{28-30}$. En suma, se analizaron un total de 27 artículos, 24 de ellos en idioma inglés y 3 en español.

\section{Resultados}

En base a la búsqueda se describen 6 tipos de cierre de fasciotomía: injerto dermoepidérmico (IDE), cierre asistido por presión negativa, cierre con elásticos vasculares en cordón de zapato o shoelace, aproximación progresiva con dispositivos de tensión estática, sutura lineal por etapas y dispositivos de dermotracción. Además, se realiza una comparación cualitativa de los estudios a través de una tabla comparativa que incluye todos los artículos obtenidos en la búsqueda en lo que respecta a tipo de estudio, tamaño muestral, outcomes, resultados y nivel de evidencia. Los resultados se detallan en la Tabla 2. 
Tabla 2. Comparación cualitativa de los estudios analizados

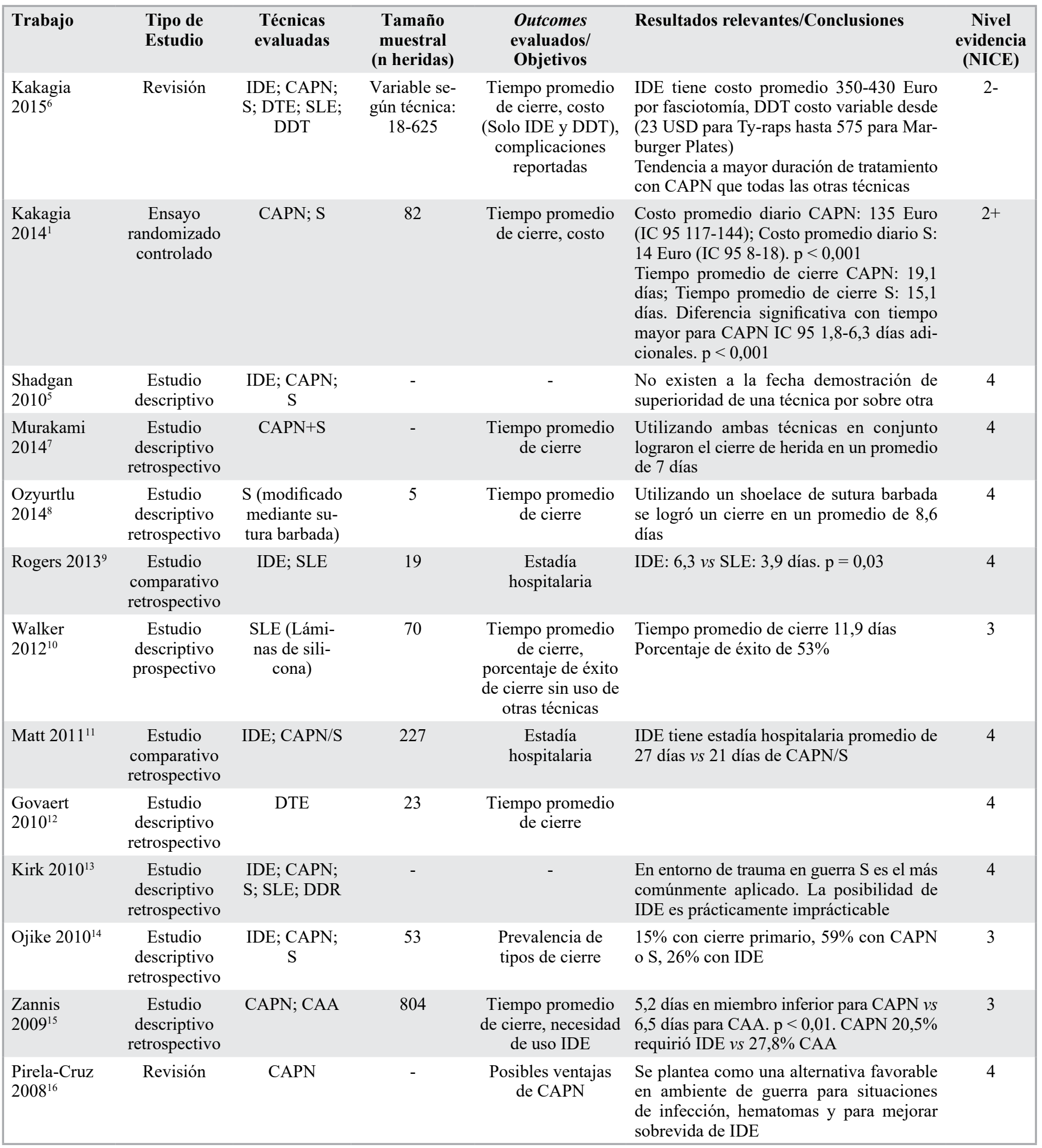


ARTíCULO DE REVISIÓN

\begin{tabular}{|c|c|c|c|c|c|c|}
\hline Trabajo & $\begin{array}{l}\text { Tipo de } \\
\text { estudio }\end{array}$ & $\begin{array}{l}\text { Técnicas } \\
\text { evaluadas }\end{array}$ & $\begin{array}{c}\text { Tamaño } \\
\text { muestral } \\
\text { (n heridas) }\end{array}$ & $\begin{array}{c}\text { Outcomes } \\
\text { evaluados/ } \\
\text { Objetivos }\end{array}$ & Resultados relevantes/Conclusiones & $\begin{array}{l}\text { Nivel } \\
\text { evidencia } \\
\text { (NICE) }\end{array}$ \\
\hline Yang $2006^{17}$ & $\begin{array}{l}\text { Estudio } \\
\text { descriptivo } \\
\text { retrospectivo }\end{array}$ & $\begin{array}{l}\text { IDE; CAPN; } \\
\quad \text { SLR }\end{array}$ & 138 & $\begin{array}{l}\text { Tiempo promedio } \\
\text { de cierre }\end{array}$ & $\begin{array}{l}\text { IDE/SLE 16,1 días vs CAPN 6,7 días } \\
\mathrm{p}<0,05\end{array}$ & 4 \\
\hline Lee $2005^{18}$ & $\begin{array}{l}\text { Reporte de } \\
\text { caso }\end{array}$ & CAPN & 1 & $\begin{array}{l}\text { Describir ventajas } \\
\text { y desventajas de } \\
\text { CAPN }\end{array}$ & $\begin{array}{l}\text { Destaca la posibilidad de maceración de } \\
\text { vasos ante exposición de estos sometidos } \\
\text { a CAPN }\end{array}$ & 4 \\
\hline $\begin{array}{l}\text { Galois } \\
2002^{20}\end{array}$ & $\begin{array}{l}\text { Reporte de } \\
\quad \text { caso }\end{array}$ & $\mathrm{S}$ modificado & 1 & $\begin{array}{l}\text { Describir una } \\
\text { modificación de } \mathrm{S}\end{array}$ & $\begin{array}{l}\text { Describe la utilización de } \mathrm{S} \text { con suturas } \\
\text { envueltas en drenajes de amplio diámetro } \\
\text { en vez de elásticos vasculares, como una } \\
\text { forma de aumentar el área de contacto con } \\
\text { músculo }\end{array}$ & 4 \\
\hline $\begin{array}{l}\text { Chiverton } \\
2000^{22}\end{array}$ & Serie de casos & $\begin{array}{l}\mathrm{S} \\
\text { (modificado) }\end{array}$ & 8 & $\begin{array}{l}\text { Describir una } \\
\text { técnica de SLE }\end{array}$ & $\begin{array}{l}\text { Se puede hacer un cierre progresivo de } \\
\text { fasciotomía mediante el uso de prolene } \\
\text { subcuticular }\end{array}$ & 4 \\
\hline $\begin{array}{l}\text { McKenney } \\
1996^{23}\end{array}$ & Revisión & DDT & - & $\begin{array}{l}\text { Tiempo promedio } \\
\text { de cierre }\end{array}$ & $\begin{array}{l}\text { Se describe que el DDT STAR tendría un } \\
\text { tiempo promedio de cierre de } 2 \text { a } 4 \text { días }\end{array}$ & 4 \\
\hline $\begin{array}{l}\text { Narayanan } \\
1996^{24}\end{array}$ & Revisión & DDT & 4 & $\begin{array}{l}\text { Describir técnica } \\
\text { de cierre de } \\
\text { fasciotomía con } \\
\text { dispositivo DDT } \\
\text { Sure-Closure }\end{array}$ & $\begin{array}{l}\text { El Sure-Closure permite en un solo tiempo } \\
\text { quirúrgico realizar aproximación suficiente } \\
\text { de los bordes de la fasciotomía para permi- } \\
\text { tir un cierre primario }\end{array}$ & 4 \\
\hline $\begin{array}{l}\text { Fleischmann } \\
1996^{25}\end{array}$ & $\begin{array}{l}\text { Estudio } \\
\text { descriptivo } \\
\text { retrospectivo }\end{array}$ & IDE; SLE & 25 & $\begin{array}{l}\text { Describir técnicas } \\
\text { demostradas de } \\
\text { cierre fasciotomía. }\end{array}$ & $\begin{array}{l}\text { Primeras descripciones de técnicas me- } \\
\text { diante IDE y SLE, describe estas opciones } \\
\text { como técnicas viables y plantea la necrosis } \\
\text { cutánea como una posible complicación }\end{array}$ & 4 \\
\hline $\begin{array}{l}\text { Kalyani } \\
2011^{27}\end{array}$ & $\begin{array}{l}\text { Revisión } \\
\text { sistemática }\end{array}$ & IDE; SLE & 34 & $\begin{array}{l}\text { Prevalencia de } \\
\text { técnica utilizada } \\
\text { para cierre }\end{array}$ & $\begin{array}{l}61 \% \text { de los casos revisados se resolvieron } \\
\text { con IDE, } 39 \% \text { con SLE }\end{array}$ & $2-$ \\
\hline $\begin{array}{l}\text { Marine } \\
2009^{28}\end{array}$ & Revisión & $\mathrm{S}$ & - & $\begin{array}{l}\text { Descripción de } \\
\text { técnica adecuada } \\
\text { de cierre de } \\
\text { fasciotomía con } \\
\text { técnica } S\end{array}$ & $\begin{array}{l}\text { S constituye una alternativa de bajo costo, } \\
\text { fácil realización, rápido aprendizaje y rá- } \\
\text { pida ejecución }\end{array}$ & 4 \\
\hline $\begin{array}{l}\text { Taladriz } \\
2014^{29}\end{array}$ & $\begin{array}{l}\text { Estudio } \\
\text { descriptivo } \\
\text { prospectivo } \\
\text { multicéntrico }\end{array}$ & $\mathrm{S}$ & 122 & $\begin{array}{l}\text { Tiempo promedio } \\
\text { de cierre }\end{array}$ & $\begin{array}{l}\text { S permite un tiempo promedio de cierre de } \\
\text { fasciotomía de } 7,9 \text { días }\end{array}$ & 3 \\
\hline Reyes $2010^{30}$ & $\begin{array}{l}\text { Estudio } \\
\text { descriptivo } \\
\text { prospectivo }\end{array}$ & $\mathrm{S}$ & 24 & $\begin{array}{l}\text { Tiempo promedio } \\
\text { de cierre, Tiempo } \\
\text { promedio de } \\
\text { estadía hospitalaria }\end{array}$ & $\begin{array}{l}\text { S permite un tiempo promedio de cierre de } \\
\text { fasciotomía de } 9,5 \text { días (DS } 3,31 \text { días) } \\
\text { S tiene una estadía hospitaliaria promedio } \\
\text { de } 12,38 \text { días (DS } 4,33 \text { días) }\end{array}$ & 3 \\
\hline
\end{tabular}

IDE: Injerto dermoepidérmico; CAPN: Cierre asistido por presión negativa; S: Shoelace; DTE: Dispositivos de tensión estática; SLE: Sutura lineal por Etapas; DDT: Dispositivos de dermotracción; CAA: Curación con apósitos activos; IC 95: intervalo confianza 95\%; DS: desviación estándar. 


\section{Injerto dermoepidérmico (IDE)}

Consiste en la obtención de una porción de tejido dermoepidérmico de grosor variable, que se escinde por completo de su fuente de irrigación en su sitio donante, para posteriormente ser transferido y fijado al sitio receptor con el fin de cubrir el defecto generado por la fasciotomía (Figura 2). Entre las ventajas encontramos las siguientes: pueden ser utilizados en heridas con bordes dañados o friables que no soportan las fuerzas de tensión esenciales para la dermotracción, permiten una cobertura precoz en heridas con gran brecha y pueden ser utilizados tanto como para cierre definitivo como a modo de terapia puente (con o sin resección del injerto inicial) hacia otro tipo de cierre definitivo, como lo es por ejemplo, el cierre primario ${ }^{6}$. Como desventaja, se destaca principalmente la morbilidad del sitio donante que constituye una nueva herida y, por ende, una nueva fuente de eventuales complicaciones para el paciente. En general, es una opción razonable para alcanzar el cierre defintivo de un defecto residual posterior a la desaparición del edema o como primera opción cuando no existe otra alterantiva o se desea acelerar el alta del paciente.

\section{Cierre asistido por presión negativa}

Consiste en la utilización de un dispositivo de aspiración que consta de 3 partes: esponja, sello y tubo aspirativo. Entre las ventajas se describen la disminución del riesgo de infección, se facilita el drenaje del edema, mejora el flujo sanguíneo local, favorece la angiogénesis y acelera el cierre de la herida $^{15,31}$. Su desventaja es el costo asociado a los insumos del dispositivo comercial (Renasys ${ }^{\circledR}$, Smith \& Nephew; Pico ${ }^{\circledR}$, Smith \& Nephew; VAC ${ }^{\circledR}, \mathrm{KCI}$ ) $\mathrm{y}$ el hecho que tiende a ser una terapia de puente para disminuir el diámetro de la fasciotomía, pero posteriormente, requieren de otras alternativas de cierre complementarias, tales como injerto ${ }^{11}$. Una de las complicaciones más graves, aunque muy poco frecuente del uso de este dispositivo es la aspiración sobre hemorragias activas lo que puede llevar incluso a la exanguinación ${ }^{32}$. Se recomienda su uso a un máximo de $100 \mathrm{mmHg}^{6}$.

\section{Cierre con elásticos vasculares en cordón de zapato o shoelace}

Técnica que consiste en el entrecruzamiento de elásticos vasculares, sujetos mediante corchetes fijos a la piel de los bordes de la herida, logrando una tensión constante que permitirá progresivamente ir ajustando los elásticos y generando una tensión lineal suficiente entre los bordes (Figura 3). En la técnica original descrita por Cohn se deben ajustar

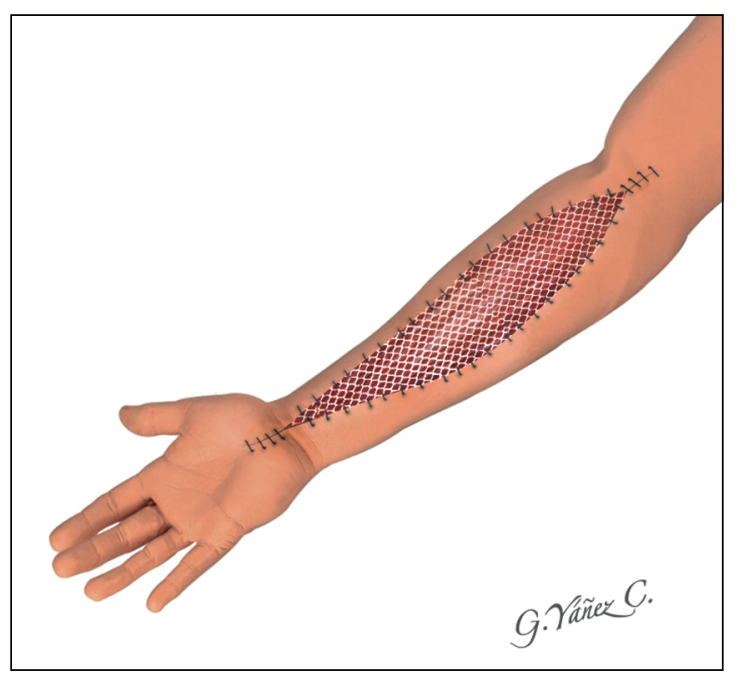

Figura 2. Injerto dermoepidérmico.

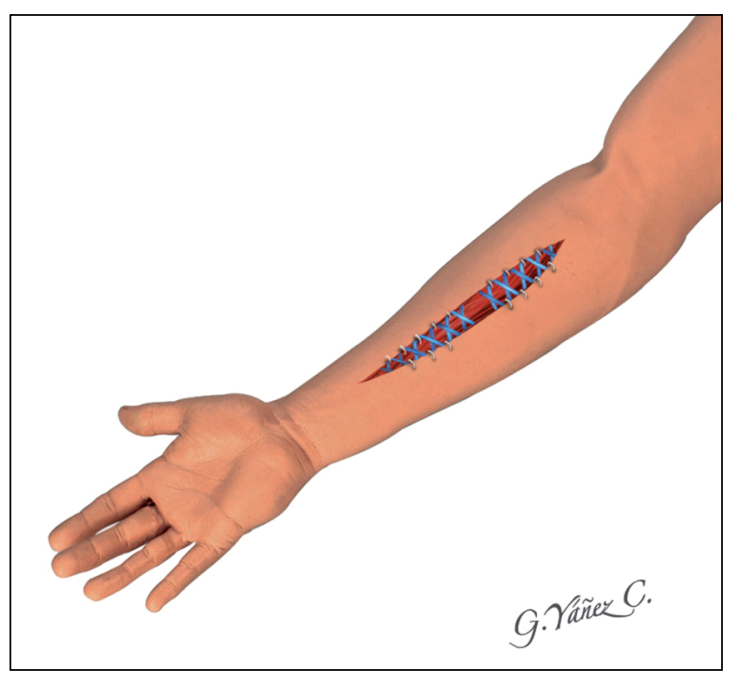

Figura 3. Shoelace: Técnica de cierre con elásticos vasculares en cordón de zapato.

los elásticos cada $48 \mathrm{~h}$, hasta aproximar los bordes y permitir la sutura lineal de la herida ${ }^{6,28-30}$. Entre las ventajas de este método podemos encontrar su bajo costo y fácil aplicación, además, de permitir observar directamente el estado de la herida y los tejidos subyacentes. Entre las desventajas destaca que la tensión producida sobre cada corchete puede generar extrusión de estos, desarrollar cierto grado de isquemia en los bordes de la herida y necesidad de un segundo procedimiento quirúrgico para el cierre de la herida ${ }^{6}$. Existen algunas variaciones de la técnica como la utilización de suturas de prolene intracuticulares que se van ajustando y permitirían un cierre directo de la herida sin necesidad de una segunda intervención ${ }^{22}$. También se describe el reemplazo de los elásticos vasculares por suturas barbadas $^{8}$. 


\section{Aproximación progresiva con dispositivos de tensión estática}

Consiste en utilizar una tela cutánea adhesiva de tensión estática, por ejemplo, Steri-strips ${ }^{\circledR}(3 \mathrm{M})$, a modo de puente entre borde de la herida. Una vez lograda la aproximación parcial de bordes se pueden añadir más adhesivos entre los previamente posicionados y posteriormente ir reemplazando aquellos que ya han perdido su fuerza tensil, así como también, añadir más puentes hacia distal para ir progresivamente cerrando la herida. La ventaja de esta alternativa es, sin duda, su bajo costo y fácil aplicación, sin embargo, no puede ser aplicada en heridas que tengan una brecha importante entre sus bordes, ya que los materiales no poseen la suficiente resistencia para hacer de puente entre éstas, además tiende a generar una cicatriz patológica y/o inestética $^{6}$.

También se describe la utilización de una lámina de silicona única que cubre la herida y se fija a los bordes de piel mediante corchetes o suturas. Posteriormente se van aproximando los bordes mediante suturas de plicatura sobre la lámina de silicona ${ }^{10}$.

\section{Sutura lineal por etapas}

Es una alternativa similar al procedimiento descrito para los dispositivos de tensión estática, pero utilizando puntos simples de sutura. Se aproximan los ángulos de la herida mediante puntos simples, por ejemplo, de Nylon 3-0, afrontando parcialmente la herida desde los extremos de manera que no se genere tensión excesiva sobre los bordes. Una vez afrontados los extremos se avanza aproximando la herida en las zonas de mayor tensión hasta conseguir un cierre lineal total. Entre las ventajas se describen su bajo costo y baja complejidad de la técnica. Sin embargo, al afrontar los bordes bajo tensión se tiende a desarrollar una cicatriz inestética e inestable. ${ }^{6}$ Dentro de las variaciones para esta técnica se describe la utilización de puntos tipo near-near-far-far ${ }^{19}$ para disminuir la tensión generada sobre los bordes.

\section{Dispositivos de dermotracción}

Consiste en la colocación de dispositivos en los bordes de la herida, los cuales mediante ajuste manual o por autoajuste generan aproximación progresiva de los bordes de la herida. Existen varios tipos de dispositivos en el mercado. A modo de ejemplo, se encuentra el dispositivo STAR $^{\circledR}$ (Suture Tension Adjustment Reel), Sure-Closure ${ }^{\circledR}$ y los TY-Raps ${ }^{\circledR}$. En el primero, en uno de los bordes de la herida se instala un cilindro rígido fijo enrollado en hilos de sutura, los cuales están unidos a un dispositivo fijo del otro borde de la herida (Figura 4). Mediante una herramienta especial se puede aumentar la tensión de los hilos entre los dispositivos, de modo que los bordes se aproximen al enrollar los hilos sobre el cilindro ${ }^{23}$. La segunda, consiste en un dispositivo plástico que consta de dos partes que se anclan a cada borde de la herida. Las mitades del dispositivo se encuentran unidas por un cilindro guía que permite, mediante ajuste manual, la aproximación de éstos, traccionando, en consecuencia, la piel subyacente sobre la cual se insertan ${ }^{6}$. El tercer ejemplo, utiliza un dispositivo (TY-Raps ${ }^{\circledR}$ ) comúnmente utilizado para procesos de embalaje, conocido coloquialmente con el nombre de "precinto", este consiste en un hilo plástico con múltiples topes, que en su extremo distal posee una ranura para el hilo, el cual una vez ensamblado no retrocede. Se debe esterilizar este dispositivo y luego fijarlo a la piel mediante corchetes $^{12}$. Dentro de las ventajas de estos dispositivos se describen su instalación en el mismo acto quirúrgico de realización de la fasciotomía para, posteriormente, ser manejados en la misma cama del paciente hasta lograr el cierre definitivo de la herida. Además, no limitan la movilización del paciente y podrían ser utilizados de forma ambulatoria. Como desventaja, son de alto costo (exceptuando los TYRaps $\left.^{\circledR}\right)$, se han descrito casos de necrosis de piel, dehiscencia de la herida, dolor, infección, recurrencia del SCA y cicatriz hipertrófica. ${ }^{6}$ Se recomienda que de optar por estos dispositivos, sean colocados en el intraoperatorio justo posterior a la fasciotomía pero que la aplicación de la tensión se postergue hasta que disminuya el edema de la extremidad. ${ }^{6}$

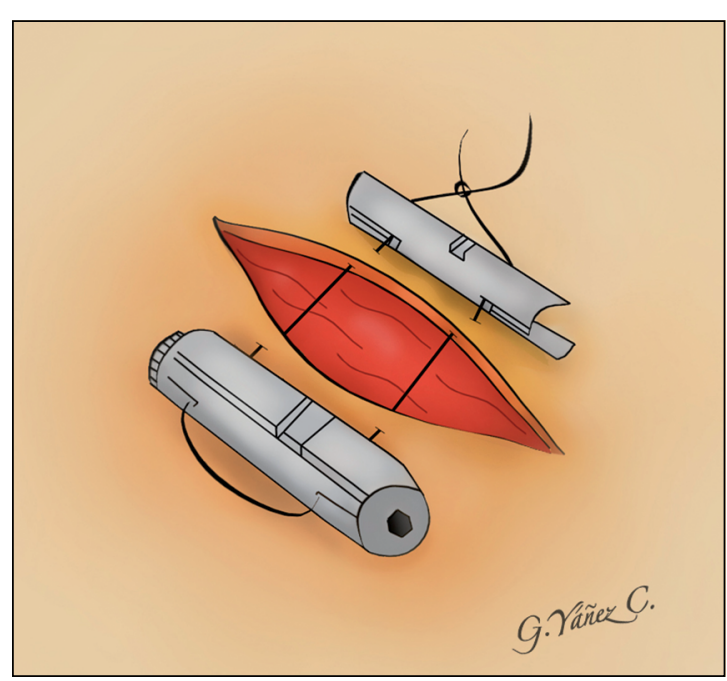

Figura 4. Dispositivo de dermotracción: Colocación del dispositivo en los bordes de la herida. Mediante ajuste manual o por autoajuste se genera aproximación progresiva de los bordes. 
Tabla 3. Ventajas y desventajas de las diferentes técnicas de cierre

\begin{tabular}{|c|c|c|}
\hline Técnica & Ventajas & Desventajas \\
\hline Injerto dermoepidérmico & $\begin{array}{l}\text { Bajo costo, aplicable a heridas con bordes dañados o } \\
\text { friables } \\
\text { Heridas con gran brecha entre bordes, edema impor- } \\
\text { tante }\end{array}$ & $\begin{array}{l}\text { Morbilidad del sitio donante (infección, profundización } \\
\text { y resultado inestético) }\end{array}$ \\
\hline $\begin{array}{l}\text { Cierre asistido por presión } \\
\text { negativa }\end{array}$ & $\begin{array}{l}\text { Disminuye el edema, mejora el flujo sanguíneo local, } \\
\text { favorece la angiogénesis y acelera el cierre de la herida }\end{array}$ & Costo, requiere ser complementado con otra técnica \\
\hline Adhesivos de tensión estática & Bajo costo, fácil aplicación & Solo es utilizable en heridas pequeñas \\
\hline Sutura lineal por etapas & Bajo costo, fácil aplicación & Resultado inestético \\
\hline $\begin{array}{l}\text { Dispositivos de dermo- } \\
\text { tracción }\end{array}$ & Movilización del paciente, uso ambulatorio & $\begin{array}{l}\text { Alto costo, riesgo de necrosis cutánea, dehiscencia } \\
\text { de la herida, dolor, infección, recurrencia del SCA y } \\
\text { cicatriz hipertrófica }\end{array}$ \\
\hline
\end{tabular}

Cabe destacar, que no existen suficientes estudios externos a las empresas fabricantes que avalen su costo-efectividad.

\section{Discusión}

Descartando el cierre por segunda intención/ curación con apósitos activos debido al pobre resultado reportado en términos de tiempo promedio de cierre y necesidad de posterior $\operatorname{IDE}^{15,26}$, es que se han descrito a la fecha al menos 6 alternativas de cierre posterior a una fasciotomía con diversos estudios acerca de su tiempo promedio de cierre, costo y necesidad de reintervención, entre otros. Cada una de estas alternativas tiene sus propias ventajas y desventajas, sin embargo, para evaluar la superioridad de una técnica sobre otra contamos mayoritariamente con estudios descriptivos de técnicas únicas, estudios comparativos entre 2 técnicas específicas o bien revisiones sistemáticas con alto grado de heterogeneidad y que evaluan outcomes variables (Tabla 2). Con ello, en la actualidad sólo se puede hacer un ánalisis individual de cada técnica, caracterizando las ventajas y desventajas de cada una (Tabla 3) de manera tal que, el cirujano en conjunto con el paciente, puedan elegir la mejor ópcion para el cierre de fasciotomía.

En la práctica, los autores recomendamos que frente a heridas muy extensas se debe evitar utilizar dispositivos de tensión estática debido a que sus materiales no poseen la suficiente resistencia y tienden a generar una cicatriz patológica y/o inestética. En caso de heridas con bordes irregulares, muy traumatizados o friables y con una gran brecha pero con un adecuado lecho receptor es mejor utilizar un IDE, considerando siempre dentro de las variables la morbilidad del sitio donante. En caso de heridas con mucho edema, siempre y cuando estén los recursos disponibles, una buena opción es asistir el cierre con un sistema de presión negativa como terapia puente al cierre definitivo con el objetivo de disminuir el edema y acelerar el cierre. Si se busca una técnica que permita cerrar la herida de forma controlada y con observación directa de los tejidos, de bajo costo y fácil de aplicar, creemos que es de mucha utilidad la técnica shoelace, sin embargo, se debe advertir la necesidad de un segundo tiempo para cierre definitivo. Los dispositivos de dermotracción si bien permiten ser utilizados de forma ambulatoria, consideramos que son costosos y engorrosos en su aplicación, pueden asociarse a necrosis de piel y cicatrización patológica por lo que no lo consideramos una técnica de primera línea. Una excepción dentro de estos dispositivos por su bajo costo, es la utilizacion de TY-Raps ${ }^{\circledR}$, sin embargo, sólo se mencionan en un solo trabajo por lo que se requieren más estudios para evaluar su efectividad.

Otra variable importante de considerar en la elección del tipo de cierre es la familiaridad del cirujano con la técnica quirúrgica, dado que al utilizar una alternativa sin la debida experiencia se puede generar un cierre deficiente de la herida, recidiva del SCA o cicatrización funcional y estéticamente deficiente.

A modo de conclusión, la heterogeneidad de las técnicas disponibles, imposibilita una adecuada revisión sistemática o metaanálisis con los trabajos publicados y es imperativo en el futuro realizar 
un estudio clínico randomizado y controlado o, al menos, un estudio comparativo que incluya las 6 técnicas de cierre mencionadas. Idealmente este diseño randomizado y prospectivo debe evaluar bajo contextos similares en heridas de fasciotomía en extremidades, todos los outcomes relevantes, como son, el tiempo promedio en alcanzar el cierre definitivo, tiempo de estadía hospitalaria, costo de la intervención, número de cirugías requeridas para alcanzar el cierre, recidiva de SCA, necesidad de extensión de fasciotomía o nueva fasciotomía, morbimortalidad, calidad de la cicatriz, uso de técnicas complementarias y calidad de vida del paciente, entre otros.

\section{Responsabilidades éticas}

Protección de personas y animales. Los autores declaran que para esta investigación no se han realizado experimentos en seres humanos ni en animales.

Confidencialidad de los datos. Los autores declaran que en este artículo no aparecen datos de pacientes.

Derecho a la privacidad y consentimiento informado. Los autores declaran que en este artículo no aparecen datos de pacientes.

\section{Financiación}

Los autores declaran no haber recibido ninguna financiación para la realización de este trabajo.

\section{Conflicto de intereses}

Los autores declaran no tener ningún conflicto de intereses.

\section{Bibliografía}

1. Kakagia D, Karadimas EJ, Drosos G, Ververidis A, Trypsiannis G, Verettas D. Wound closure of leg fasciotomy: comparison of vacuum-assisted closure versus shoelace technique. A randomised study. Injury. 2014;45(5):890-3.

2. McQueen MM, Gaston P, Court-Brown CM. Acute compartment syndrome. Who is at risk? J Bone Joint Surg Br 2000; 82: 200-3.

3. Via AG, Oliva F, Spoliti M, Maffulli N. Acute compartment syndrome. Muscles, Ligaments and Tendons Journal. 2015;5(1):18-22.

4. Schwartz JT, Brumback RJ, Lakatos R, Poka A, Bathon GH, Burgess AR. Acute compartment syndrome of the thigh: a spectrum of injury. J Bone Joint Surg Am. 1989;71:392-400.

5. Shadgan B, Menon M, Sanders D, Berry G, Martin C Jr, Duffy P, et al. Current thinking about acute compartment syndrome of the lower extremity. Can J Surg. 2010;53(5):329-34.

6. Kakagia D. How to Close a Limb Fasciotomy Wound: An Overview of Current Techniques. Int J Low Extrem Wounds. 2015;14(3):268-76.

7. Murakami M, Morikage N, Samura M, Yamashita O, Suehiro K, Hamano K.
Shoelace technique plus vacuum-assisted closure in leg fasciotomy. ANZ J Surg. 2014;84(10):795.

8. Ozyurtlu M, Altınkaya S, Baltu Y, Ozgenel GY. A new, simple technique for gradual primary closure of fasciotomy wounds. Ulus Travma Acil Cerrahi Derg. 2014;20(3):194-8.

9. Rogers GF, Maclellan RA, Liu AS, Taghinia AH, Labow BI, Meara JG et al. Extremity fasciotomy wound closure: comparison of skin grafting to staged linear closure. J Plast Reconstr Aesthet Surg. 2013;66(3):90-1.

10. Walker T, Gruler M, Ziemer G, Bail DH. The use of a silicon sheet for gradual wound closure after fasciotomy. J Vasc Surg. 2012;55(6):1826-8.

11. Matt SE, Johnson LS, Shupp JW, Kheirbek T, Sava JA. Management of fasciotomy wounds: Does the dressing matter? Am Surg. 2011;77(12):1656-60.

12. Govaert GA, van Helden S. Ty-raps in trauma: a novel closing technique of extremity fasciotomy wounds. J Trauma. 2010;69(4):972-5.

13. Kirk KL, Hayda R. Compartment syndrome and lower-limb fasciotomies in the combat environment. Foot Ankle Clin. 2010;15(1):41-61.

14. Ojike NI, Roberts CS, Giannoudis PV.
Compartment syndrome of the thigh: a systematic review. Injury. 2010;41(2):1336.

15. Zannis J, Angobaldo J, Marks M, DeFranzo A, David L, Molnar J et al. Comparison of fasciotomy wound closures using traditional dressing changes and the vacuum-assisted closure device. Ann Plast Surg. 2009;62(4):407-9.

16. Pirela-Cruz MA, Machen MS, Esquivel D. Management of large soft-tissue wounds with negative pressure therapylessons learned from the war zone. J Hand Ther.2008;21(2):196-202.

17. Yang CC, Chang DS, Webb LX. Vacuumassisted closure for fasciotomy wounds following compartment syndrome of the leg. J Surg Orthop Adv. 2006;15(1):19-23.

18. Lee AT, Fanton GS, McAdams TR. Acute compartment syndrome of the thigh in a football athlete: a case report and the role of the vacuum-assisted wound closure dressing. J Orthop Trauma. 2005;19(10):748-50.

19. Dahners LE. The running near-nearfar-far stitch for closure of fasciotomies and other large wounds. Orthopedics. 2003;26(4):383-4.

20. Galois L, Pauchot J, Pfeffer F, Kermarrec I, Traversari R, Mainard D, et al. Modified shoelace technique for delayed 
primary closure of the thigh after acute compartment syndrome. Acta Orthop Belg. 2002;68(1):63-7.

21. Janzing HM, Broos PL. Dermatotraction: an effective technique for the closure of fasciotomy wounds: a preliminary report of fifteen patients. J Orthop Trauma. 2001;15(6):438-41.

22. Chiverton N, Redden JF. A new technique for delayed primary closure of fasciotomy wounds. Injury. 2000;31(1):21-4.

23. McKenney MG, Nir I, Fee T, Martin L, Lentz K. A simple device for closure of fasciotomy wounds. Am J Surg. 1996;172(3):275-7.

24. Narayanan K, Latenser BA, Jones LM, Stofman G. Simultaneous primary closure of four fasciotomy wounds in a single setting using the Sure-Closure device. Injury. 1996;27(6):449-51.

25. Fleischmann W, Lang E, Kinzl L. Vacuum assisted wound closure after dermatofasciotomy of the lower extremity. Unfallchirurg. 1996;99(4):283-7.

26. Walker M, Kralik D, Porritt K. Fasciotomy wounds associated with acute compartment syndrome: a systematic review of effective treatment. JBI Database of Systematic Reviews \& Implementation Report. 2014;12(1):101175.

27. Kalyani BS, Fisher BE, Roberts CS, Giannoudis PV. Compartment syndrome of the forearm: a systematic review. The Journal of hand surgery. 2011;36(3):53543.

28. Mariné L. Técnica de cierre diferido de incisiones de fasciotomías con elásticos. Rev Chil Cir. 2009;61(2):203-205. Disponible en: www.scielo.cl (Consultado el 29 de enero de 2017).

29. Taladriz C, Reyes A, Derosas Ca, Farías J, Siegel S, Torres J y cols. Cierre primario de fasciotomías con técnica con elásticos: experiencia nacional multicéntrica. Rev Chil Cir. 2014; 66(5): 423-428. Disponible en: www.scielo.cl (Consultado el 29 de enero de 2017).

30. Reyes A, Siegel S, Torres J, Derosas C, Acuña $\mathrm{F}$ y cols. Afrontamiento primario con elásticos vasculares en el manejo del cierre progresivo de fasciotomías. Rev Chil Cir. 2010: 62(4):377-381. Disponible en: www.scielo.cl (Consultado el 29 de enero de 2017).

31. Kakagia D, Karadimas E, Drosos G, Ververidis A, Kazakos D, Lazarides M et al. Vacuum-assisted closure downgrades reconstructive demands in high-risk patients with severe lower extremity injuries. Acta Chir Plast. 2009;51:59-64.

32. White R,Miki R, Kazmier P, Anglen JO. Vacuum-assisted closure complicated by erosion and hemorrhage of the anterior tibial artery. J Orthop Trauma. 2005;19:56-59. 